\title{
Mass-scaling replica-exchange molecular dynamics optimizes computational resources with simpler algorithm
}

\author{
Tetsuro NAGA and Takuya TAKAHASH b) \\ Department of Bioinformatics, School of Life Sciences, Ritsumeikan University, Kusatsu, Siga 525-85077, \\ Japan
}

(Dated: 12 April 2021)

We develop a novel method of replica-exchange molecular dynamics (REMD) simulation, mass-scaling REMD (MSREMD) method, which improves trajectory accuracy at high temperatures, and thereby contributes to numerical stability. In addition, the MSREMD method can also simplify a replica-exchange routine by eliminating velocity scaling. As a pilot system, a Lennard-Jones fluid is simulated with the new method. The results show that the MSREMD method improves the trajectory accuracy at high temperatures compared with the conventional REMD method. We analytically demonstrate that the MSREMD simulations can reproduce completely the same trajectories of the conventional REMD ones with shorter time steps at high temperatures in case of the Nosé-Hoover thermostats. Accordingly, we can easily compare the computational costs of the REMD and MSREMD simulations. We conclude that the MSREMD method decreases the instability and optimizes the computational resources with simpler algorithm under the constant trajectory accuracy at all temperatures.

PACS numbers: 05.20.-y,02.70.Ns,05.10.Ln

Keywords: generalized-ensemble algorithm, replica-exchange method (REM), parallel tempering, replicaexchange molecular dynamics (REMD), mass scaling, Nosé-Hoover thermostat, numerical stability

\section{INTRODUCTION}

Monte Carlo (MC) and molecular dynamics (MD) simulations have been widely applied to many systems in the computational statistical physics field. However, the quasi-ergodicity problem, where simulations are prone to get trapped in states of energy local-minima, has been a great difficulty. In order to conquer this difficulty, generalized-ensemble algorithms have been developed and applied to many systems including spin systems and biomolecular systems (for reviews, see, e.g., Refs. 13).

Commonly practiced examples of the generalizedensemble algorithms are the multicanonical (MUCA) algorithm,$\frac{4,5}{}$, the simulated tempering $\frac{6,7}{,}$, and the replicaexchange method (REM) ${ }^{8}, 9$ (it is also referred to as the parallel tempering). Closely related to MUCA are the Wang-Landau algorithm 10.11 and metadynamics 12 . Also closely related to REM is the method in Ref. 13, which is later detailed in Ref. 14. The REM was first involved with MC simulations, and later the idea was also applied to MD simulations. The replica-exchange molecular dynamics (REMD) 15 method is the MD version of REM. Note that there are a number of attempts to generalize the REM and REMD, such as multi-dimensional extensions (see e.g., Refs. 16 20) including the NPT ensemble ${ }^{3.21}-23$ and the combination of the Tsallis statistics 24 with REM (see, e.g., Ref. 25).

In this work, we particularly focus on a practical con-

\footnotetext{
a) tnagai@fc.ritsumei.ac.jp

b) tkhs@sk.ritsumei.ac.jp
}

cern in application of the REMD method, in order to improve its efficiency. As the temperature increases, trajectory accuracy of simulations decreases and the simulations become numerically instable. Generally speaking, the shorter time step is necessary for the higher temperatures. However, this is not elegantly taken account of in previous applications. The same time step is usually employed for all replicas. Some take risks of using a time step validated at low temperature for all replicas; others prudently employ a too short time step at low temperatures. For example, one of the authors has applied the REMD method to lipid bilayer systems with a coarsegrained model 26,27 , and chose a shorter time step than that suggested in Ref. 26, to avoid the trajectory inaccuracy at high temperatures 28,29 . Such difficulty is also depicted in Ref. 30, in which they employed a long time step for their force field, the Protein in Atomistic details coupled with Coarse-grained Environment (PACE), combing a united-atom and a coarse-grained force field. However, they tripled the mass of all proteins in the REMD simulations in order to avoid crashes at a high temperature. We believe that there should be a number of preliminary simulations unpublished because such trajectory inaccuracy due to the usage of a long time step at high temperatures causes some numerical instability.

One common practice for ensuring the accuracy and enhancing sampling efficiency is mass-scaling method which focused on increasing mass of hydrogen atom ${ }^{31,32}$. For details including the historical aspect, see Ref. 32 and the references therein. The heavier mass enables one to use the larger time step with the same accuracy. This method is especially useful for quantummechanics/molecular-mechanics (QM/MM) simulations where covalent bond constraint algorithms such as 
SHAKE ${ }^{33}$ RATTLE 34 are not suitable $\frac{35}{3}$, because chemical reactions involve bond forming and breaking. Note that there are other attempts of scaling masses to enhance the sampling efficiency ${ }^{36}-38$.

In this article, using the idea mentioned above, we present a mass-scaling REMD (MSREMD) method, where masses of all particles are scaled according to the reference temperature assigned to each replica. The heavier particles at the higher reference temperatures improve the trajectory accuracy of simulations. Furthermore, the MSREMD method does not require the velocity scaling necessary for the conventional REMD method and thereby the algorithm is simpler.

This article is organized as follows. In section II we shall briefly review the REMD method and introduce the MSREMD method. We prove that mass scaling in the equations of motion with the Nosé-Hoover thermostat $\underline{39,40}$ is equivalent to the change of time step. Section III is devoted to Results and Discussion. We show that the MSREMD method restores the trajectory accuracy at the high temperatures. After we compare physical quantities obtained with the REMD and MSREMD methods, we contrast the estimated computational costs of the REMD simulation with a long time step supposed to be verified at the lowest temperature, the REMD simulation with a short time step supposed to be validated at the highest temperature, and the MSREMD simulation. We conclude this paper in section IV with some outlook.

\section{MODEL AND METHODS}

\section{A. Review of the REMD method}

Before we present the MSREMD method, we shall simply review the REMD method. Readers who would like to know details about REM and REMD are referred to, e.g., Refs. 8, 15, 41 43. Because the MSREMD method follows the multi-dimensional REM formalization, we review the REMD method following Ref. 16 .

We consider a system consisting of $N$ particles, of which coordinate and momentum vectors are given by $q=\left\{\boldsymbol{q}_{1}, \ldots, \boldsymbol{q}_{N}\right\}$ and $p=\left\{\boldsymbol{p}_{1}, \ldots, \boldsymbol{p}_{N}\right\}$, respectively. The velocity vector is denoted by $\dot{q}=\left\{\dot{\boldsymbol{q}}_{1}, \ldots, \dot{\boldsymbol{q}}_{N}\right\}$, where the dot stands for the time derivative. The kinetic energy and the potential energy are denoted by $K$ and $E$, respectively, with its total energy $H=K+E$. In this work, we assume $\boldsymbol{p}_{k} / m_{k}=\dot{\boldsymbol{q}}_{k}$ for $k=1,2, \ldots, N$, where $m_{k}$ denotes the mass of the $k$ th particle. The kinetic energy is therefore given by

$$
K(\dot{q})=\sum_{k}^{N} \frac{m_{k} \dot{\boldsymbol{q}}_{k}^{2}}{2}
$$

For the convenience in introducing the MSREMD method, we use $q$ and $\dot{q}$ instead of $q$ and $p$. In the canonical ensemble at the reference temperature $T$, the state $\{q, \dot{q}\}$ is weighted by the Boltzmann factor,

$$
f_{\mathrm{NVT}}(q, \dot{q}) \propto \exp \left[-H(q, \dot{q}) / k_{\mathrm{B}} T\right] .
$$

In the REMD method, $N_{\text {rep }}$ copies of systems, namely replica 1 , replica $2, \ldots$, and replica $N_{\text {rep }}$ are simulated at the same condition except for the reference temperatures. Each replica is coupled to exclusively one of the $N_{\text {rep }}$ different temperatures denoted by $T_{1}, T_{2}, \ldots, T_{N_{\text {rep }}}$. For simplicity, we assume $T_{1}<T_{2}<\cdots<T_{N_{\text {rep }}}$. Every certain MD steps $N_{\text {ex }}$, replicas attempt to exchange their reference temperatures. These exchanges of the temperatures cause replicas to perform a random walk in the temperature space, and this in turn induces a random walk in the energy space. The random walk of energy helps systems to overcome the energetic barriers.

In order to look into the REMD method further, we let replica $i$ be assigned to $T_{l}$. Due to its one-to-one correspondence, the replica index $i\left(\in\left\{1,2, \ldots, N_{\text {rep }}\right\}\right)$ is given by the permutation function of the temperature label $l\left(\in\left\{1,2, \ldots, N_{\text {rep }}\right\}\right)$ and vice versa. We thus have

$$
\begin{aligned}
& i=i(l)=l^{-1}(l), \\
& l=l(i)=i^{-1}(i) .
\end{aligned}
$$

The replica-exchange attempts are judged by Metropolis criterion ${ }^{44}$, of which base is on the detailed balance condition; the replica-exchange attempt between $T_{l}$ and $T_{m}$ is accepted at the probability of

$$
\min [1, \exp (\Delta \beta \Delta E)]
$$

where $\Delta \beta=1 /\left(k_{\mathrm{B}} T_{l}\right)-1 /\left(k_{\mathrm{B}} T_{m}\right)$ and $\Delta E=E_{i_{\text {old }}(l)}-$ $E_{i^{\text {old }}(m)}$ with $k_{\mathrm{B}}$ the Boltzmann constant and $E_{i^{\text {old }}(l)}$ the potential energy of the replica coupling to $T_{l}$ before the replica-exchange attempt. Although the Metropolis criterion for replica-exchange attempts is used, the Gibbs sampler $\underline{45}$ and the Suwa-Todo method $\underline{46,47}$ are also applicable.

If the replica-exchange attempt is accepted, $i(l)$ will be reassigned:

$$
\begin{aligned}
& i^{\text {new }}(l)=i^{\text {old }}(m), \\
& i^{\text {new }}(m)=i^{\text {old }}(l),
\end{aligned}
$$

where the superscripts of old and new express before and after the accepted replica-exchange attempt, respectively. After the exchange, the velocities are requested to be uniformly scaled in the manner of

$$
\begin{gathered}
\dot{q}_{i^{\text {new }(l)}}^{\prime}=\sqrt{\frac{T_{l}}{T_{m}}} \dot{q}_{i_{\text {new }}(l)}=\sqrt{\frac{T_{l}}{T_{m}}} \dot{q}_{i^{\text {old }}(m)}, \\
\dot{q}_{i^{\text {new }(m)}}^{\prime}=\sqrt{\frac{T_{m}}{T_{l}}} \dot{q}_{i^{\text {new }}(m)}=\sqrt{\frac{T_{m}}{T_{l}}} \dot{q}_{i^{\text {old }}(l)},
\end{gathered}
$$

where $\dot{q}_{i^{\text {new }}(l)}^{\prime}$ stands for the velocity vector after the velocity scaling. The theoretical basis for using such modified states is explicitly discussed in Ref. 48. The scaling of 
the velocities is required because the kinetic energy distributions and reference temperatures are different from a replica to another.

When a constant-temperature MD simulation involve a deterministic thermostat with extra variables, a treatment particular to the thermostat is necessary to meet the detailed balance condition $\underline{43}$. The Nosé-Hoover thermostat $\underline{39}, \underline{40}$ must be one of the most common thermostats for constant-temperature simulations, and we focus on the thermostat. Assuming ergodicity, the NoséHoover thermostat with the reference temperature $T$ realizes the probability density function of $\{q, \dot{q}, \eta\}$ :

$$
\begin{aligned}
& f_{\mathrm{NH}}(q, \dot{q}, \eta) \\
& \propto \exp \left[-\left(\sum_{k}^{N} \frac{m_{k} \dot{\boldsymbol{q}}_{k}^{2}}{2}+E(q)+Q \eta^{2} / 2\right) / k_{\mathrm{B}} T\right] \\
& =\exp \left[-H(q, \dot{q}) / k_{\mathrm{B}} T\right] \exp \left[-Q \eta^{2} / 2 k_{\mathrm{B}} T\right],
\end{aligned}
$$

where $\eta$ and $Q$ stand for the rate and mass of NoséHoover thermostat, respectively. We again use the velocity instead of the momentum for the later convenience. Integrating the probability density function with respect to $\eta$, one obtains the canonical distribution with regards to $\{q, \dot{q}\}$.

Let us recall that the REMD method is based on the detailed balance condition. When the Nosé-Hoover thermostat is employed for REMD simulations, the detailed balance condition must be imposed considering $\eta$ as well as $\{q, \dot{q}\}$. It is shown particularly in Ref. 43 that $\eta$ is also requested to be scaled similarly to the velocity. In practical, the detailed balance condition is fulfilled by setting $Q \propto T$ without scaling of $\eta$, which is implicitly practiced in, e.g., GROMACS software package $\underline{\underline{49}} \underline{\underline{52}}$.

We shall explore the probability density function of a system studied with the REMD method involving the Nosé-Hoover thermostats. We let the Greek letter $\xi_{i(l)}$ stand for the state of the replica coupling to $T_{l}: \xi_{i(l)}=$ $\left\{q_{i(l)}, \dot{q}_{i(l)}, \eta_{i(l)}\right\}$. Because the replicas are noninteracting in the REMD method, the state $X$ can be identified by specifying all of the replicas: $X=\left\{\xi_{i(1)}, \ldots, \xi_{i\left(N_{\text {rep }}\right)}\right\}$. Hence the REMD simulation with the Nosé-Hoover thermostat has the probability density function of

$$
\Pi_{\mathrm{REMD}}(X)=\Pi_{\mathrm{REMD}}\left(\xi_{i(1)}, \ldots, \xi_{i\left(N_{\mathrm{rep}}\right)}\right)=\prod_{l} f_{\mathrm{NH}}\left(\xi_{i(l)}\right)
$$

$\propto \prod_{l} \exp \left[-\left(\sum_{k}^{N} \frac{m_{k} \dot{\boldsymbol{q}}_{k, i(l)}^{2}}{2}+E\left(q_{i(l)}\right)+Q \eta_{i(l)}^{2} / 2\right) / k_{\mathrm{B}} T_{l}\right]$.

\section{B. MSREMD method}

We now formalize the MSREMD method with the Nosé-Hoover thermostat. Note that the formalization is applicable to other thermostats such as Langevin ${ }^{53}$ and Andersen ${ }^{54}$ ones by eliminating the terms originating from the Nosé-Hoover thermostat. Because the Hamiltonian is not necessarily identical among the replicas ${ }^{16}$, we choose the masses of all particles uniformly proportional to the reference temperature. Substituting $m_{k}=m_{0, k} T_{l} / T_{1}$ as well as $Q=Q_{0} k_{\mathrm{B}} T_{l}$ for the NoséHoover thermostat to Eq. 14, we obtain

$$
\begin{aligned}
& \Pi_{\operatorname{MSREMd}}(X) \propto \\
& \prod_{l} \exp \left[-\left(\sum_{k}^{N} \frac{m_{0, k} \dot{\boldsymbol{q}}_{k, i(l)}^{2}}{2 k_{\mathrm{B}} T_{1}}+Q_{0} \eta_{i(l)}^{2} / 2\right)\right] \\
& \times \exp \left[-E\left(q_{i(l)}\right) / k_{\mathrm{B}} T_{l}\right],
\end{aligned}
$$

which signifies that the velocity vector $\dot{q}_{i(l)}$ shares the identical distribution among all of the reference temperatures and so does the Nosé-Hoover thermostat rate $\eta_{i(l)}$. Thus we can exchange the velocities and rates of the Nosé-Hoover thermostat without the care such as the Metropolis criterion or velocity scaling. On the other hand, the coordinate vectors can be exchanged according to the probability given by Eq. 5] as in the REMD method. Moreover, the scaled masses enable one to restore the trajectory accuracy of simulation at high temperatures. Note that the exchange of the velocities instead of the momenta is crucial for this algorithm, because the momenta have different probability density functions among the reference temperatures.

We explicitly show that the Metropolis criterion given by Eq. [5 can be used for the replica-exchange attempts in the MSREMD simulation without the velocity scaling. Letting $X^{\text {old }}$ and $X^{\text {new }}$ be

$$
X^{\text {old }}=\left\{\xi_{i(1)}, \ldots, \xi_{i^{\text {old }}(l)}, \ldots, \xi_{i^{\text {old }}(m)}, \ldots, \xi_{i\left(N_{\text {rep }}\right)}\right\}
$$

and

$$
X^{\text {new }}=\left\{\xi_{i(1)}, \ldots, \xi_{i^{\text {new }}(l)}, \ldots, \xi_{i^{\text {new }}(m)}, \ldots, \xi_{i\left(N_{\text {rep }}\right)}\right\},
$$

respectively, we look into the ratio of transition probabilities under the detailed balance condition between these two states, say $w\left(X^{\text {old }} \rightarrow X^{\text {new }}\right)$ and $w\left(X^{\text {new }} \rightarrow X^{\text {old }}\right)$. Using Eqs. 6] and 7, we obtain 


$$
\begin{aligned}
& \frac{w\left(X^{\text {old }} \rightarrow X^{\text {new }}\right)}{w\left(X^{\text {new }} \rightarrow X^{\text {old }}\right)} \\
& =\exp \left[-\left(\sum_{k}^{N} \frac{m_{0, k} \dot{\boldsymbol{q}}_{k, i^{\text {new }}(l)}^{2}}{2 k_{\mathrm{B}} T_{1}}+Q_{0} \eta_{i^{\text {new }}(l)}^{2} / 2\right)\right] \exp \left[-E\left(q_{i^{\text {new }}(l)}\right) / k_{\mathrm{B}} T_{l}\right] \\
& \times \exp \left[-\left(\sum_{k}^{N} \frac{m_{0, k} \dot{\boldsymbol{q}}_{k, i^{\mathrm{new}}(m)}^{2}}{2 k_{\mathrm{B}} T_{1}}+Q_{0} \eta_{i^{\text {new }}(m)}^{2} / 2\right)\right] \exp \left[-E\left(q_{i^{\mathrm{new}}(m)}\right) / k_{\mathrm{B}} T_{m}\right] \\
& /\left(\exp \left[-\left(\sum_{k}^{N} \frac{m_{0, k} \dot{\boldsymbol{q}}_{k, i^{\text {old }}(l)}^{2}}{2 k_{\mathrm{B}} T_{1}}+Q_{0} \eta_{i^{\text {old }}(l)}^{2} / 2\right)\right] \exp \left[-E\left(q_{i^{\text {old }}(l)}\right) / k_{\mathrm{B}} T_{l}\right]\right. \\
& \left.\times \exp \left[-\left(\sum_{k}^{N} \frac{m_{0, k} \dot{\boldsymbol{q}}_{k, i^{\text {old }}(m)}^{2}}{2 k_{\mathrm{B}} T_{1}}+Q_{0} \eta_{i^{\text {old }}(m)}^{2} / 2\right)\right] \exp \left[-E\left(q_{i^{\text {old }}(m)}\right) / k_{\mathrm{B}} T_{m}\right]\right) \\
& =\exp \left[\left(E\left(q_{i^{\text {old }}(l)}\right)-E\left(q_{i^{\text {old }}(m)}\right)\right)\left(1 / k_{\mathrm{B}} T_{l}-1 / k_{\mathrm{B}} T_{m}\right)\right] \text {. }
\end{aligned}
$$

In this way, we obtain the same term of $\Delta \beta \Delta T$ in Eq. 5 .

To wrap up, MSREMD simulations with Nosé-Hoover thermostat can be performed as follows: (1) prepare $N_{\text {rep }}$ replicas with the masses of all of the particles and the Nosé-Hoover thermostats being in proportion to the reference temperature; (2) perform the $N_{\text {rep }}$ independent canonical MD simulations at each temperature; (3) exchange the replicas according to the probability given by Eq. 5 without any velocity scaling; (4) go back to the step (2).

\section{Equations of motion of mass-scaled system}

We analytically demonstrate that mass scaling in the equation of motion of the Nosé-Hoover thermostat 39,40 is mathematically identical to changing the time step. The equations of motion are given by

$$
\begin{aligned}
\dot{q} & =\frac{p}{m}, \\
\dot{p} & =F(q)-\eta p, \\
\dot{\eta} & =\frac{p^{2} / m-3 N k_{\mathrm{B}} T}{Q},
\end{aligned}
$$

where $F$ denotes force. For simplicity we set $m_{1}=m_{2}=$ $\cdots=m_{N} \equiv m$. We consider the transformations given by

$$
\begin{aligned}
m & =\alpha m^{\prime} \\
t & =\sqrt{\alpha} t^{\prime} \\
Q & =\alpha Q^{\prime} \\
q & =q^{\prime} \\
\eta & =\frac{1}{\sqrt{\alpha}} \eta^{\prime},
\end{aligned}
$$

where $\alpha$ denotes the scaling factor. Substituting these transformations into Eqs. 1921] we obtain

$$
\begin{aligned}
\frac{d q^{\prime}}{d t^{\prime}} & =\frac{p^{\prime}}{m^{\prime}}, \\
\frac{d p^{\prime}}{d t^{\prime}} & =F\left(q^{\prime}\right)-\eta^{\prime} p^{\prime}, \\
\frac{d \eta^{\prime}}{d t^{\prime}} & =\frac{p^{2} / m^{\prime}-3 N k_{\mathrm{B}} T}{Q^{\prime}},
\end{aligned}
$$

with $p^{\prime}=p / \sqrt{\alpha}$. Therefore the time step $\Delta t$ of the system with the mass value $m$ correspond to the time step $\Delta t^{\prime}=\Delta t / \sqrt{\alpha}$ of the system with the mass value $m^{\prime}=m / \alpha$.

We assume the primed quantities to be normal REMD ones at $T_{l}$, and the scaling factor $\alpha$ is $T_{l} / T_{1}$. The MSREMD simulation at $T_{l}$ with the time step $\Delta t$ generate the same time evolution of coordinate vector as the REMD simulation with the time step at $T_{l}$,

$$
\Delta t^{\prime}(l)=\frac{\Delta t}{\sqrt{T_{l} / T_{1}}} .
$$

On the other hand, the evolution of the momentum (or velocity) vector and rate of Nosé-Hoover thermostat is reproduced by using the scaling factor.

\section{Models}

We employed a Lennard-Jones (LJ) fluid as a handful pilot system. The potential energy is given by

$$
\begin{aligned}
E & =\sum_{i<j} V_{i j} \\
V_{i j} & =4 \epsilon\left[\left(\frac{\sigma}{r_{i j}}\right)^{12}-\left(\frac{\sigma}{r_{i j}}\right)^{6}\right],
\end{aligned}
$$


where $\epsilon$ and $\sigma$ represent the value of the potential minimum and the diameter of particle, respectively, and $r_{i j}$ the distance between the $i$ th and $j$ th particles. Hereafter, we use reduced units; we set $\sigma=1, \epsilon=1$, and $k_{\mathrm{B}}=1$.

\section{E. Numerical details}

The integrator was an in-house program. We used the time reversible integrator $\underline{55}$. The choice of Suzuki-Trotter decomposition was made following Ref. 56. This choice corresponds to Integrator 1 in Ref. 57. As a pseudorandom number generator for the replica-exchange routine, the Mersenne twister $\frac{58}{}$ was employed.

We performed both the REMD and MSREMD simulations. Three time steps $\Delta t$ were employed: $\Delta t=0.002$, 0.005 , and 0.01 for each method. The total numbers of MD steps were $10^{6}, 4 \times 10^{5}$, and $2 \times 10^{5}$ with the total time length fixed at 2000 . The number of replica was eight $\left(N_{\text {rep }}=8\right)$ and the reference temperatures were $1.000,1.104,1.219,1.346,1.486,1.641,1.812$ and 2.000 . The exchange acceptance rates ranged from $14 \%$ to $19 \%$ in all the simulations. Identical five-hundred LJ particles were placed $(N=500)$ in a cube of which side was 8.55 in the reduced length unit, corresponding to the number density $\rho=0.800$. In these thermal conditions, the LJ fluid is in the liquid phase 5 . The periodic boundary condition was employed.

The particle mass was set to unity for all of the replicas in the REMD simulations. On the other hand, the mass was given by $T_{l} / T_{1}$ for the replica coupling to $T_{l}$ in the MSREMD simulations. The mass of the Nosé-Hoover thermostat was set to $Q_{0}=10$ for the MSREMD and REMD simulations. The replica-exchange attempts were made every $N_{\text {ex }}=10$ steps. At the MD step of $N_{\text {ex }} \times 2 n$ for $n=1,2,3 \ldots$, the replica-exchange attempts were made between $T_{1}$ and $T_{2}$, between $T_{3}$ and $T_{4}$, between $T_{5}$ and $T_{6}$, and between $T_{7}$ and $T_{8}$. Correspondingly, at the MD step of $N_{\mathrm{ex}} \times(2 n-1)$, the replica-exchange attempts were made between $T_{2}$ and $T_{3}$, between $T_{4}$ and $T_{5}$, and between $T_{6}$ and $T_{7}$. The LJ forces were simply truncated at $3\left(r_{\mathrm{c}}=3\right)$ in the reduced unit, and accordingly the LJ potential was shifted upward by $\left|V_{i j}\left(r_{\mathrm{c}}\right)\right|$ for $r_{i j}<r_{\mathrm{c}}$.

\section{F. Evaluation of Trajectory Accuracy}

We evaluated the simulation inaccuracy through the fluctuation of the conservation energy. The conservation energy of the Nosé-Hoover MD simulation with the reference temperature $T_{\text {ref }}$ is given by

$$
\begin{aligned}
& E_{\text {cons }}(t)= \\
& \qquad \sum_{k}^{N} \frac{\boldsymbol{p}_{k}^{2}}{2 m_{k}}+E(q)+\frac{1}{2} Q \eta^{2}+3 N k_{\mathrm{B}} T_{\mathrm{ref}} \int_{0}^{t} \eta\left(t^{\prime}\right) d t^{\prime} .
\end{aligned}
$$

Practically, this quantity fluctuates reflecting numerical errors. Thus the sum of absolute fluctuation per unit time is used for the evaluation of the trajectory accuracy:

$$
\Delta E_{\text {cons }} \equiv \frac{1}{\Delta t N_{\text {step }}} \sum_{i=1}^{N_{\text {step }}}\left|E_{\text {cons }}(i \Delta t)-E_{\text {cons }}((i-1) \Delta t)\right|,
$$

where $N_{\text {step }}$ is the number of MD steps. Note that the way of evaluation is similar to Eq. 74 in Ref. [55, which is given by

$$
\frac{1}{N_{\text {rep }}} \sum_{i=1}^{N_{\text {step }}}\left|\frac{E_{\text {cons }}(i \Delta t)-E_{\text {cons }}(0)}{E_{\text {cons }}(0)}\right| .
$$

We used the deviations in the conservation energy between the successive time steps because $E_{\text {cons }}(0)$ changes due to the accepted replica-exchange attempts. We checked the relationship between $\log \Delta t$ and $\log \Delta E_{\text {cons }}$ and confirmed that the gradient values were 2.0 and almost agree with those in Ref. 55 .

\section{RESULTS AND DISCUSSION}

Figure 1 shows the probability density functions of the velocity $x$-component, $v_{x}$, obtained with the REMD and MSREMD simulations for $\Delta t=0.01$. We took account of all of the particles. The velocities at the half time steps were used in the calculation of the kinetic energy according to the recommendation in Ref. 57 . The probability density functions were different among the temperatures in the REMD simulation, whereas those obtained in the MSREMD simulations were the same among the temperatures. These results show that the proper scaling of mass enables one to produce the same probability density function of velocity.

Figure 2 shows the trajectory inaccuracy of the simulations, as measured by $\Delta E_{\text {cons }}$, with an inset of the logarithmic ordinate. The REMD simulations become more inaccurate as the temperature increases. In contrast, the trajectory accuracy at high temperatures obtained with the MSREMD simulations is of the same level as the low temperatures for each time step. The slight increases in the trajectory inaccuracy with regards to the temperature in the MSREMD simulation could be attributed to the steeper potential surfaces faced at higher potential energy values. Therefore, the trajectory inaccuracy at high temperature can be substantially reduced by the MSREMD method, which infers that one can perform more numerically stable simulations with the MSREMD method.

Table I shows the average potential and kinetic energies obtained with the REMD and MSREMD simulations for all of the reference temperatures. The errors in Table \were obtained by using the jackknife method $\underline{42,60,61}$ with twenty bins. Figure 3 shows the radial distribution function for $T_{1}, T_{4}$ and $T_{8}$ obtained with the two 


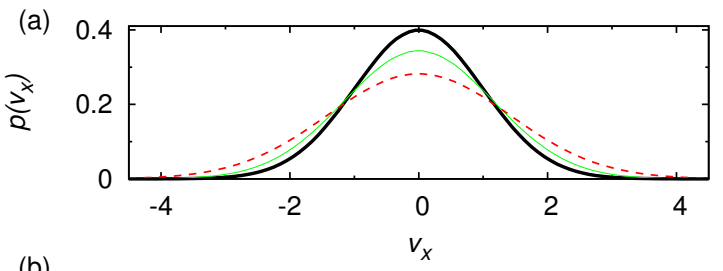

(b)

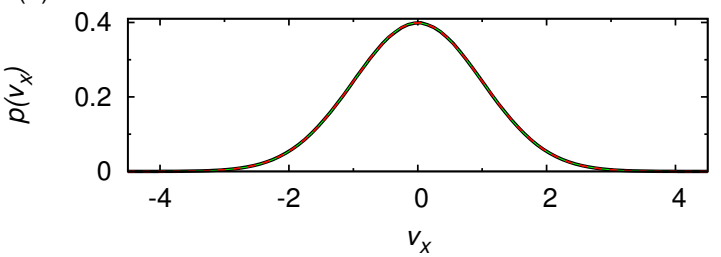

FIG. 1. Probability density functions of $v_{x}, p\left(v_{x}\right)$, for (a) REMD and (b) MSREMD simulations. Black thick, green thin, and red dashed lines represent the functions for $T_{1}, T_{4}$ and $T_{8}$, respectively. Those at other temperatures are suppressed to aid the eye. Lines completely overlap in MSREMD simulation $[$ see $(b)]$.

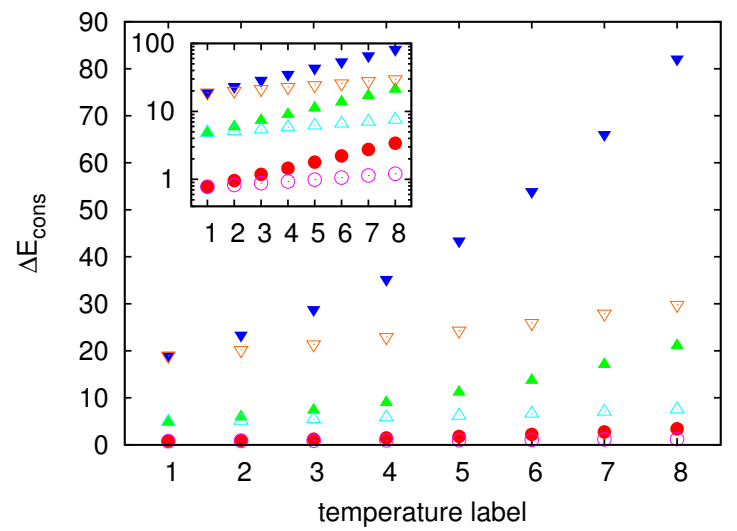

FIG. 2. Trajectory accuracy $\Delta E_{\text {cons }}$ plotted against temperature label. Red closed circles, green closed triangles and blue closed inversed triangles represent the results of normal REMD simulations for $\Delta t=0.002,0.005$ and 0.01 , respectively. Magenta open circles, cyan open triangles and orange open inversed triangles show the results of MSREMD simulations for $\Delta t=0.002,0.005$ and 0.01 , respectively. Error bars are so small that they are suppressed to aid the eye. The inset is the same plot with the logarithm ordinate.

methods. These results show the very good agreement between the two methods, which is very natural consequences. All of the coordinate-related quantities must be the same between the two methods because the changes of masses do not affect the configurational partition function. In addition, the same amount of the kinetic energy ought to be distributed to each degree of freedom at the same temperature regardless of the weight of particles, due to the equipartition theorem in the classical statistical physics. Note that we did not find appreciable difference in the sizes of errors between the two methods (see
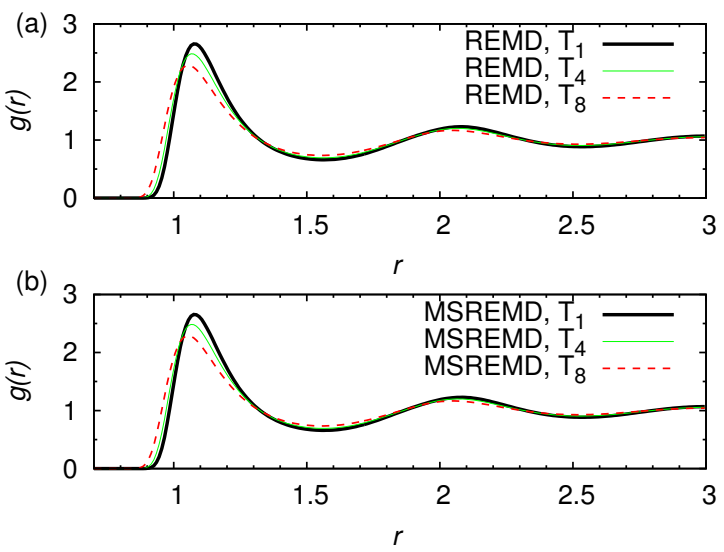

FIG. 3. Radial distribution functions $g$ for (a) the normal REMD and (b) MSREMD simulations, as a function of distance $r$ between particles. Black thick, green thin, and red dashed lines represent replica 1, 4 and 8, respectively. The other replicas are suppressed to aid the eye.

Table 【).

We compare the computational costs of the three simulations: the long-time-step REMD (LTS-REMD) simulation of which time step $\Delta t_{\mathrm{L}}$ is validated at the lowest temperature; the short-time-step REMD (STS-REMD) simulation with the time step $\Delta t_{\mathrm{S}}=\sqrt{T_{1} / T_{N_{\mathrm{rep}}}} \Delta t_{\mathrm{L}}$ prudently validated at the highest temperature; and the MSREMD simulation with the time step $\Delta t_{\mathrm{L}}$.

As a measure of the computational cost, we calculate the efficiency ratio of the STS-REMD simulation to the LTS-REMD simulation, $f_{\mathrm{S}}$, as follows. Because the time step is given by $\Delta t_{\mathrm{S}}=\sqrt{T_{1} / T_{N_{\mathrm{rep}}}} \Delta t_{\mathrm{L}}$, the trajectory obtained with the STS-REMD simulation is $\sqrt{T_{1} / T_{N_{\text {rep }}}}$ times as long as that obtained with the LTS-REMD simulation, which yields the efficiency ratio,

$$
f_{\mathrm{S}}(Z)=\sqrt{\frac{T_{1}}{T_{N \text { rep }}}}=\frac{1}{\sqrt{Z}}<1,
$$

where a temperature ratio $Z$ is $T_{N_{\text {rep }}} / T_{1}$. The efficiency ratio of the LTS-REMD simulation to the LTS-REMD simulation, $f_{\mathrm{L}}$ is obviously unity.

Owing to the correspondence shown in section IIC, the trajectory belonging to $T_{l}$ in the MSREMD simulation is $\sqrt{T_{1} / T_{l}}(\leq 1)$ times as long as that in the LTSREMD simulation. Consequently, the efficiency ratio of the MSREMD simulation to the LTS-REMD simulation is given by

$$
\frac{1}{N_{\text {rep }}} \sum_{l=1}^{N_{\text {rep }}} \sqrt{\frac{T_{1}}{T_{l}}} .
$$

Because the temperatures are usually given according to a geometric series, the efficiency ratio $f_{\mathrm{M}}\left(Z, N_{\text {rep }}\right)$ turns 
TABLE I. Average kinetic energy $\langle K\rangle$ and average potential energy $\langle E\rangle$ obtained with the two methods at all the reference temperatures. Kinetic energy was calculated with the velocities at half time $\operatorname{steps}^{57}$. Errors were evaluated with the jackknife method. Exact average kinetic energy obtained by $\frac{3}{2} N T$ is also given.

\begin{tabular}{lcccccc}
\hline \hline & & \multicolumn{2}{c}{ REMD } & & \multicolumn{2}{c}{ MSREMD } \\
\cline { 3 - 4 } \cline { 6 - 6 }$T_{l}$ & $\frac{3}{2} N T_{l}$ & $\langle K\rangle$ & $\langle E\rangle$ & $\langle K\rangle$ & $\langle E\rangle$ \\
\hline 1.000 & 750.00 & $750.02 \pm 0.05$ & $-2519.3 \pm 0.1$ & $750.11 \pm 0.05$ & $-2519.7 \pm 0.1$ \\
1.104 & 828.00 & $827.96 \pm 0.08$ & $-2474.1 \pm 0.2$ & $828.0 \pm 0.1$ & $-2474.5 \pm 0.2$ \\
1.219 & 914.25 & $914.34 \pm 0.09$ & $-2425.8 \pm 0.2$ & $914.1 \pm 0.1$ & $-2426.1 \pm 0.2$ \\
1.346 & 1009.5 & $1009.45 \pm 0.09$ & $-2374.2 \pm 0.2$ & & $1009.5 \pm 0.1$ & $-2373.9 \pm 0.2$ \\
1.486 & 1114.50 & $1114.5 \pm 0.1$ & $-2319.2 \pm 0.2$ & & $1114.7 \pm 0.1$ & $-2318.5 \pm 0.2$ \\
1.641 & 1230.75 & $1230.6 \pm 0.1$ & $-2259.3 \pm 0.2$ & & $1230.8 \pm 0.1$ & $-2258.9 \pm 0.2$ \\
1.812 & 1359.0 & $1359.1 \pm 0.1$ & $-2195.8 \pm 0.2$ & & $1358.9 \pm 0.1$ & $-2195.3 \pm 0.3$ \\
2.000 & 1500.0 & $1500.0 \pm 0.1$ & $-2128.1 \pm 0.2$ & & $1500.0 \pm 0.1$ & $-2128.3 \pm 0.3$ \\
\hline \hline
\end{tabular}

out to be

$$
f_{\mathrm{M}}\left(Z, N_{\text {rep }}\right)=\frac{1}{N_{\text {rep }}} \frac{1-\left(\frac{1}{\sqrt{Z}}\right)^{\frac{N_{\text {rep }}}{N_{\text {rep }}-1}}}{1-\left(\frac{1}{\sqrt{Z}}\right)^{\frac{1}{N_{\text {rep }}-1}}} .
$$

For $N_{\text {rep }} \rightarrow \infty, f_{\mathrm{M}}$ is given by

$$
f_{\mathrm{M}}(Z, \infty) \equiv \lim _{N_{\mathrm{rep}} \rightarrow \infty} f_{\mathrm{M}}\left(Z, N_{\text {rep }}\right)=\frac{1-\left(\frac{1}{\sqrt{Z}}\right)}{\ln \sqrt{Z}} .
$$

The values of $f_{\mathrm{M}}(Z, \infty)$ are $0.8451 \ldots$ and $0.7694 \ldots$ for $Z=2$ and 3 , respectively. Note that $Z=3$ corresponds to $T_{1}=300 \mathrm{~K}$ and $T_{N_{\text {rep }}}=900 \mathrm{~K}$, which should represent the popular application of the REMD method to all-atom simulations. The limit value of $f_{\mathrm{M}}(Z, \infty)$ is the lower bound as is illustrated in Figure 4, which shows how $f_{\mathrm{M}}$ converges for $Z=2$ and $Z=3$ as $N_{\text {rep }}$ tends to infinity. The convergence is fast and we use the limit values as the value of efficiency ratio of the MSREMD simulation to the LTS-REMD simulation.

Figure 5 illustrates $f_{\mathrm{L}}, f_{\mathrm{S}}$, and $f_{\mathrm{M}}$ for $N_{\text {rep }}=\infty$ as functions of $Z$. The efficiency of the STS-REMD and MSREMD simulations decrease as $Z$ increases. The MSREMD simulation is more efficient than the STSREMD simulation $\left(f_{\mathrm{S}}<f_{\mathrm{M}}\right)$. The efficiency ratio of the MSREMD simulation to the STS-REMD simulation is given by

$$
\frac{f_{\mathrm{M}}}{f_{\mathrm{S}}}=\frac{\sqrt{Z}-1}{\ln \sqrt{Z}} .
$$

The values of $f_{\mathrm{M}} / f_{\mathrm{S}}$ for $Z=2$ and $Z=3$ are $1.195 \ldots$ and $1.333 \ldots$, respectively. Therefore the MSREMD simulation is $20 \%$ to $30 \%$ more efficient than the STS-REMD simulation.

\section{CONCLUSIONS}

We introduced the MSREMD method, where we scale the masses of all the particles uniformly proportional

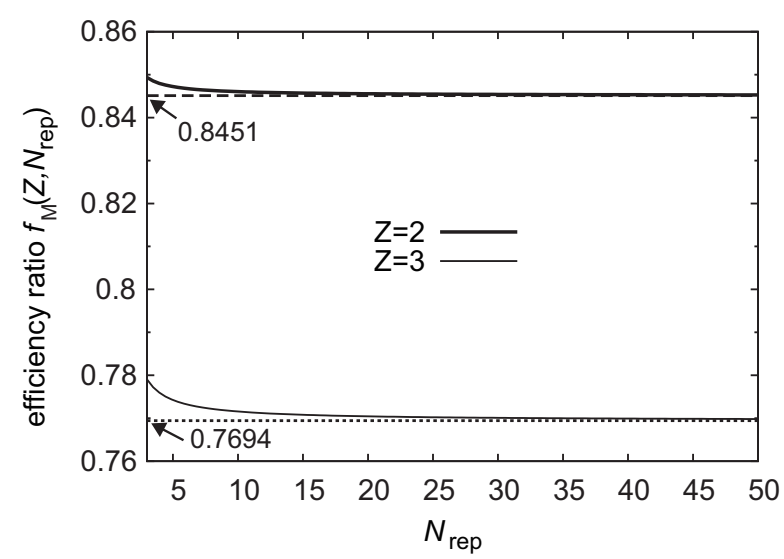

FIG. 4. Efficiency ratio $f_{\mathrm{M}}$ plotted against the number of replicas, $N_{\text {rep }}$. Thick solid, and thin solid lines represent the efficiency ratios $f_{\mathrm{M}}\left(2, N_{\text {rep }}\right)$ and $f_{\mathrm{M}}\left(3, N_{\text {rep }}\right)$, respectively. Dashed and dotted lines approximately correspond to the values of $f_{\mathrm{M}}(2, \infty)$ and $f_{\mathrm{M}}(3, \infty)$, respectively.

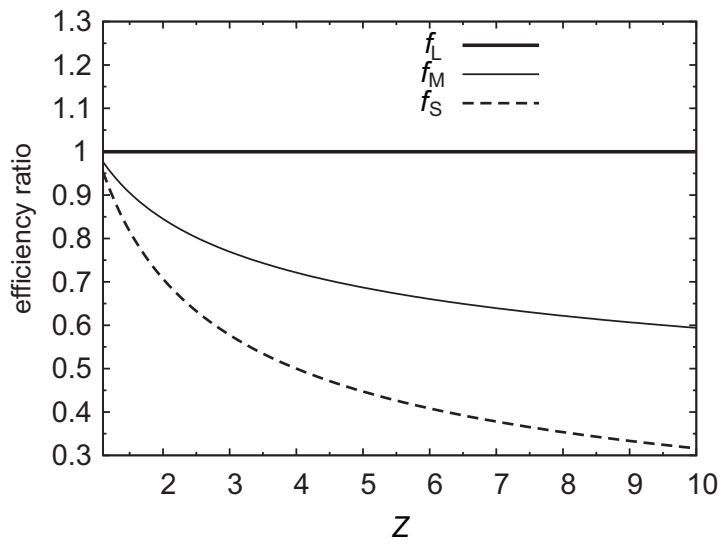

FIG. 5. Efficiency ratios $f_{\mathrm{L}}, f_{\mathrm{M}}$, and $f_{\mathrm{S}}$ as functions of the temperature ratio $Z$. Thick solid, thin solid, and dashed lines represent $f_{\mathrm{L}}(Z), f_{\mathrm{M}}(Z, \infty)$ and $f_{\mathrm{S}}(Z)$, respectively.

to the reference temperatures. We analytically showed 
that the scaling of mass in the equations of motion with the Nosé-Hoover thermostat corresponds to the scaling of time step. The larger masses at the higher reference temperatures help one restore the trajectory accuracy at the high temperatures, which infers that more stable simulations are feasible. Moreover, the identicalness of the velocity distributions realized by the MSREMD method enables one to exchange the replicas without velocity scaling, and thereby the replica-exchange routine is simpler. Because we only manipulate mass values in the MSREMD method, the coordinate-related quantities such as the radial distribution function and the average potential energy are identical to those obtained with the REMD method. The kinetic energy distributions and the heat capacities are also identical between the two methods.

We evaluated the efficiency ratios of the STS-REMD and MSREMD simulations to the LTS-REMD simulation. The MSREMD simulation should typically use $20 \%$ to $30 \%$ more resources than the LTS-REMD simulation with the potentially risky time step validated at the lowest temperature. On the other hand, the MSREMD simulation typically uses $20 \%$ to $30 \%$ less computational resources than the STS-REMD simulation with the short time step validated at high temperatures. The MSREMD method therefore balances the trajectory accuracy and the computational cost by effectively adjusting the time steps according to the reference temperatures.

One interesting extension of the MSREMD method for biomolecules would be to change the way of scaling according to the atom species as well. Such an extension would be useful for e.g. the QM/MM simulations, where the covalent bond constraint algorithms are not suitable. We also expect that the new method works well with coarse-grained models. Whereas we particularly focused on the NVT ensemble, the rigorous formalization and evaluation of the MSREMD method with other thermostats or other ensembles are our interesting future task.

\section{ACKNOWLEDGMENTS}

Some of computations were performed at the Research Center for Computational Science, Okazaki, Japan. This work was, in part, supported by Grant-in-Aid for Young Scientists (B) under Grant No. 26790083. TT gratefully acknowledges support by Grant-in-Aid for Scientific Research (C) under Grant No. 25440065 and Grant-in-Aid for Scientific Research on Innovative Areas under Grant No. 20118003.

\footnotetext{
${ }^{1}$ U. H. E. Hansmann and Y. Okamoto, "The generalized-ensemble approach for protein folding simulations," in Annual Reviews of Computational Physics VI, edited by D. Stauffer (World Scientific, Singapore, 1999) pp. 129-157.

${ }^{2}$ A. Mitsutake, Y. Sugita, and Y. Okamoto, "Generalizedensemble algorithms for molecular simulations of biopolymers." Biopolymers 60, 96-123 (2001)
}

${ }^{3}$ Y. Sugita and Y. Okamoto, "Free-Energy Calculations in Protein Folding by Generalized-Ensemble Algorithms," in Lecture Notes in Computational Science and Engineering edited by T. Schlick and H. H. Gan (Springer, 2002) pp. 304-332, http://arxiv.org/abs/cond-mat/0102296.

${ }^{4}$ B. A. Berg and T. Neuhaus, "Multicanonical algorithms for first order phase transitions," Physics Letters B 267, 249-253 (1991)

${ }^{5}$ B. A. Berg and T. Neuhaus, "Multicanonical ensemble: A new approach to simulate first-order phase transitions," Physical Review Letters 68, 9-12 (1992).

${ }^{6}$ A. P. Lyubartsev, A. A. Martsinovski, S. V. Shevkunov, and P. N. Vorontsov-Velyaminov, "New approach to Monte Carlo calculation of the free energy: Method of expanded ensembles," The Journal of Chemical Physics 96, 1776 (1992)

${ }^{7}$ E. Marinari and G. Parisi, "Simulated tempering: A new Monte Carlo scheme," Europhysics Letters (EPL) 19, 451-458 (1992)

${ }^{8}$ K. Hukushima and K. Nemoto, "Exchange Monte Carlo method and application to spin glass simulations," Journal of the Physics Society Japan 65, 1604-1608 (1996)

${ }^{9}$ C. J. Geyer, in Computing Science and Statistics, Proceedings of the 23rd Symposium on the Interface, edited by E. M. Keramidas (Interface Foundation of North America, 1991) pp. 156-163.

${ }^{10} \mathrm{~F}$. Wang and D. P. Landau, "Efficient, multiple-range random walk algorithm to calculate the density of states," Physical Review Letters 86, 2050-2053 (2001)

${ }^{11}$ F. Wang and D. P. Landau, "Determining the density of states for classical statistical models: A random walk algorithm to produce a flat histogram," Physical Review E 64, 056101 (2001)

${ }^{12}$ A. Laio and M. Parrinello, "Escaping free-energy minima," Proc. Natl. Acad. Sci. USA 99, 12562-12566 (2002)

${ }^{13}$ R. H. Swendsen and J.-S. Wang, "Replica Monte Carlo simulation of spin-glasses," Physical Review Letters 57, 2607-2609 (1986)

${ }^{14}$ J.-S. Wang and R. H. Swendsen, "Replica Monte Carlo simulation (Revisited)," Progress of Theoretical Physics Supplement 157, 317-323 (2005) ${ }^{15}$ Y. Sugita and Y. Okamoto, "Replica-exchange molecular dynamics method for protein folding," Chemical Physics Letters 314, 141-151 (1999)

${ }^{16}$ Y. Sugita, A. Kitao, and Y. Okamoto, "Multidimensional replica-exchange method for free-energy calculations," The Journal of Chemical Physics 113, 6042 (2000)

${ }^{17}$ A. Mitsutake and Y. Okamoto, "From multidimensional replica-exchange method to multidimensional multicanonical algorithm and simulated tempering," Physical Review E 79, 047701 (2009)

${ }^{18}$ A. Mitsutake and Y. Okamoto, "Multidimensional generalized-ensemble algorithms for complex systems." The Journal of Chemical Physics 130, 214105 (2009)

${ }^{19}$ A. Mitsutake, "Simulated-tempering replicaexchange method for the multidimensional version." The Journal of Chemical Physics 131, 094105 (2009).

${ }^{20}$ T. Nagai and Y. Okamoto, "Simulated tempering and magnetizing: Application of two-dimensional simulated tempering to the two-dimensional Ising model and its crossover," Physical Review E 86, 056705 (2012)

${ }^{21}$ T. Nishikawa, H. Ohtsuka, Y. Sugita, M. Mikami, and Y. Okamoto, "Replicaexchange Monte Carlo Method for Ar fluid," Progress of Theoretical Physics Supplement 138, 270-271 (2000)

${ }^{22}$ T. Okabe, M. Kawata, Y. Okamoto, and M. Mikami, "Replicaexchange Monte Carlo method for the isobaric-isothermal ensemble," Chemical Physics Letters 335, 435-439 (2001)

${ }^{23}$ D. Paschek and A. García, "Reversible Temperature and Pressure Denaturation of a Protein Fragment: A Replica Exchange Molecular Dynamics Simulation Study," Physical Review Letters 93, 10-13 (2004)

${ }^{24}$ C. Tsallis, "Possible generalization of Boltzmann-Gibbs statistics," Journal of Statistical Physics 52, 479-487 (1988) 
${ }^{25}$ J. Kim and J. E. Straub, "Generalized simulated tempering for exploring strong phase transitons," The Journal of Chemical Physics 133, 154101 (2010)

${ }^{26}$ S. J. Marrink, A. H. de Vries, and A. E. Mark, "Coarse Grained Model for Semiquantitative Lipid Simulations," The Journal of Physical Chemistry B 108, 750-760 (2004)

${ }^{27}$ S. J. Marrink, H. J. Risselada, S. Yefimov, D. P. Tieleman, and A. H. de Vries, "The MARTINI force field: coarse grained model for biomolecular simulations." The Journal of Physical Chemistry. B 111, 7812-7824 (2007)

${ }^{28}$ T. Nagai and Y. Okamoto, "Replica-exchange molecular dynamics simulation of a lipid bilayer system with a coarse-grained model," Molecular Simulation 38, 437-441 (2012)

${ }^{29}$ T. Nagai, R. Ueoka, and Y. Okamoto, "Phase Behavior of a Lipid Bilayer System Studied by a Replica-Exchange Molecular Dynamics Simulation," Journal of the Physical Society of Japan 81, 024002 (2012)

${ }^{30} \mathrm{~W}$. Han, C.-K. Wan, F. Jiang, and Y.-D. Wu, "PACE force field for protein simulations. 1. Full parameterization of version 1 and verification," Journal of Chemical Theory and Computation 6, 3373-3389 (2010)

${ }^{31}$ R. Pomès and J. McCammon, "Mass and step length optimization for the calculation of equilibrium properties by molecular dynamics simulation," Chemical Physics Letters 166, 425-428 (1990)

${ }^{32}$ K. A. Feenstra, B. Hess, and H. J. C. Berendsen, "Improving efficiency of large time-scale molecular dynamics simulations of hydrogen-rich systems," Journal of Computational Chemistry 20, 786-798 (1999).

${ }^{33}$ J.-P. Ryckaert, G. Ciccotti, and H. J. Berendsen, "Numerical integration of the cartesian equations of motion of a system with constraints: molecular dynamics of n-alkanes," Journal of Computational Physics 23, 327-341 (1977)

${ }^{34}$ H. C. Andersen, "Rattle: A "velocity" version of the shake algorithm for molecular dynamics calculations," Journal of Computational Physics 52, 24-34 (1983).

${ }^{35}$ H. Zheng, S. Wang, and Y. Zhang, "Increasing the time step with mass scaling in Born-Oppenheimer ab initio QM/MM molecular dynamics simulations." Journal of Computational Chemistry 30, 2706-2711 (2009).

${ }^{36}$ B. Mao, "Mass-weighted molecular dynamics simulation and conformational analysis of polypeptide." Biophysical journal 60, 611-22 (1991)

${ }^{37}$ B. Mao, G. M. Maggiora, and K. C. Chou, "Massweighted molecular dynamics simulation of cyclic polypeptides." Biopolymers 31, 1077-86 (1991)

${ }^{38} \mathrm{P}$. H. Nguyen, "Replica exchange simulation method using temperature and solvent viscosity." The Journal of Chemical Physics 132, 144109 (2010)

${ }^{39}$ S. Nosé, "A molecular dynamics method for simulations in the canonical ensemble," Molecular Physics 52, 255-268 (1984)

${ }^{40}$ W. Hoover, "Canonical dynamics: Equilibrium phase-space distributions," Physical Review A 31, 1695-1697 (1985)

${ }^{41}$ D. Frenkel and B. Smit, Understanding molecular simulation: from algorithms to applications (Access Online via Elsevier, 2001).

${ }^{42}$ B. A. Berg, Markov Chain Monte Carlo Simulations and Their Statistical Analysis (World Scientific, Singapore, 2004) ; http://www.worldscibooks.com/physics/5602.html

${ }^{43}$ Y. Mori and Y. Okamoto, "Replica-Exchange Molecular Dynamics Simulations for Various Constant Temperature Algorithms,"
Journal of the Physical Society of Japan 79, 074001 (2010)

${ }^{44}$ N. Metropolis, A. W. Rosenbluth, M. N. Rosenbluth, A. H. Teller, E. Teller, et al., "Equation of state calculations by fast computing machines," The Journal of Chemical Physics 21, 1087 (1953)

${ }^{45} \mathrm{~J}$. D. Chodera and M. R. Shirts, "Replica exchange and expanded ensemble simulations as gibbs sampling: Simple improvements for enhanced mixing," The Journal of Chemical Physics 135, 194110 (2011).

${ }^{46} \mathrm{H}$. Suwa and S. Todo, "Markov chain Monte Carlo method without detailed balance," Physical Review Letters 120603, 120603 (2010)

${ }^{47} \mathrm{~S}$. G. Itoh and H. Okumura, "Replica-permutation method with the Suwa-Todo algorithm beyond the replica-exchange method," Journal of Chemical Theory and Computation 9, 570-581 (2013) ${ }^{48} \mathrm{~W}$. Nadler and U. Hansmann, "Optimizing replica exchange moves for molecular dynamics," Physical Review E 76, 057102 (2007)

${ }^{49} \mathrm{H}$. Berendsen, D. van der Spoel, and R. van Drunen, "GROMACS: A message-passing parallel molecular dynamics implementation," Computer Physics Communications 91, 43-56 (1995)

${ }^{50}$ E. Lindahl, B. Hess, and D. van der Spoel, "GROMACS 3.0: a package for molecular simulation and trajectory analysis," Journal of Molecular Modeling, 306-317 (2001)

${ }^{51}$ D. Van Der Spoel, E. Lindahl, B. Hess, G. Groenhof, A. E. Mark, and H. J. C. Berendsen, "GROMACS: fast, flexible, and free." Journal of Computational Chemistry 26, 1701-1718 (2005)

${ }^{52}$ S. Pronk, S. Páll, R. Schulz, P. Larsson, P. Bjelkmar, R. Apostolov, M. R. Shirts, J. C. Smith, P. M. Kasson, D. van der Spoel, B. Hess, and E. Lindahl, "GROMACS 4.5: a high-throughput and highly parallel open source molecular simulation toolkit," Bioinformatics 29, 845-854 (2013).

${ }^{53} \mathrm{M}$. Allen and D. Tildesley, Computer simulation of liquids (Oxford : Clarendon Press, New York, 1989).

${ }^{54} \mathrm{H}$. C. Andersen, "Molecular dynamics simulations at constant pressure and/or temperature," The Journal of Chemical Physics 72, $2384(1980)$.

${ }^{55}$ G. J. Martyna, M. E. Tuckerman, D. J. Tobias, and M. L. Klein, "Explicit reversible integrators for extended systems dynamics," Molecular Physics 87, 1117-1157 (1996).

${ }^{56}$ H. Okumura, S. G. Itoh, and Y. Okamoto, "Explicit symplectic integrators of molecular dynamics algorithms for rigid-body molecules in the canonical, isobaric-isothermal, and related ensembles." The Journal of chemical physics 126, 084103 (2007)

${ }^{57}$ S. G. Itoh, T. Morishita, and H. Okumura, "Decomposition-order effects of time integrator on ensemble averages for the Nosé-Hoover thermostat," The Journal of Chemical Physics 139, 064103 (2013).

${ }^{58}$ M. Matsumoto and T. Nishimura, "Mersenne twister: a 623dimensionally equidistributed uniform pseudo-random number generator," ACM Transactions on Modeling and Computer Simulation (TOMACS) 8, 3-30 (1998).

${ }^{59} \mathrm{H}$. Okumura and F. Yonezawa, "Liquid-vapor coexistence curves of several interatomic model potentials," The Journal of Chemical Physics 113, 9162 (2000)

${ }^{60} \mathrm{~B}$. Efron, The Jackknife, the Bootstrap, and Other Resampling Plans (Society for Industrial and Applied Mathmatics [SIAM], Philadelphia, 1982).

${ }^{61} \mathrm{H}$. Flyvbjerg and H. G. Petersen, "Error estimates on averages of correlated data," The Journal of Chemical Physics 91, 461-466 (1989) 